\title{
Article
}

\section{Retraction of Rod-like and Spheroidal Droplets and Stress Relaxation after Step Shear Strain in Polymer Blends}

\author{
Masaoki TAKAHASHI $^{*}$ and Kenzo OKAMOTO** \\ ${ }^{*}$ Department of Macromolecular Science and Engineering, Kyoto Institute of Technology, \\ Matsugasaki, Sakyo-Ku, Kyoto 606-8585, Japan \\ ${ }^{* *}$ Venture Laboratory, Kyoto Institute of Technology, Matsugasaki, Sakyo-Ku, Kyoto 606-8585, Japan \\ (Received : March 12, 2007)
}

\begin{abstract}
After application of a large step shear strain, a polymer droplet in an immiscible polymer matrix takes rod-like and spheroidal shapes before returning to the spherical shape. Change in semi axes of those droplets is calculated based on the Cohen-Carriere theory and the extension of the theory by Okamoto et al. From comparison with experimental data, it has been found that retraction of semi axes is well described by the theory using a hydrodynamic factor for the droplet associated with the viscous resistance of the matrix. The excess shear stress for rod-like and spheroidal droplets is predicted based on the Doi-Ohta theory by evaluating the interface tensor from the semi axes calculated. The predicted excess shear stress for the deformed droplet is close to experimental data of a polymer blend with narrow distribution of droplet size after normalization per one droplet with the volume-averaged radius. The effects of polydispersity and interaction with adjacent droplets in the blend are suggested for the remaining difference between the prediction and the data.
\end{abstract}

Key Words: Polymer blend / Droplet phase / Stress relaxation / Doi-Ohta theory / Cohen-Carriere theory

\section{INTRODUCTION}

In polymer blends, prediction on interface shape and excess shear stress under shear flow is very important to control the blend morphology and to find better processing condition. However, the prediction is still very difficult even if we limit our consideration to immiscible blend systems with droplet/ matrix structure. In the present study, droplet shape and excess shear stress are considered in the simplest case, i.e., retraction of a droplet under a step shear strain, to concentrate on the effect of interfacial tension. In our previous studies on a poly(isobutylene) (PIB)/poly(dimethyl siloxane) (PDMS) system $^{1,2)}$ we found that the interface shape of droplet observed at large step shear strains changes as: flat ellipsoid, rod-like shape, dumbbell, spheroid, and finally sphere. From the calculation of interface area of each shape, it was revealed that the droplet shape should change in this order to reduce the interfacial free energy. ${ }^{1,2)}$ Recently, it was found that the amplitude of Rayleigh instability in the rod-like period is so small and that the dumbbell shape appears due to the endpinching mechanism. ${ }^{3)}$

In our previous study on measurement of interfacial tension $^{4)}$, the theory of Cohen and Carriere ${ }^{5)}$ on retraction of a rod-like droplet in a quiescent matrix was extended to a spheroidal droplet. This improved method of imbedded fiber retraction was applied to polystyrene/poly(methyl methacrylate) melt systems ${ }^{4)}$ and to PIB/PDMS systems. ${ }^{6)}$ It was shown that the improved method gives comparable value of interfacial tension with those obtained by the breaking thread and pendant drop methods.

For immiscible binary blends, a basic equation relating the shape of interface and the excess stress tensor $\Delta \sigma$ due to the anisotropy of interface was first presented by Batchelor ${ }^{7}$, and was later reformulated by Onuki ${ }^{8,9}$ and Doi and Ohta. ${ }^{10)}$

$$
\Delta \boldsymbol{\sigma}=-\Gamma \mathbf{q}=-\frac{\Gamma}{V} \int d S\left(\mathbf{n n}-\frac{1}{3} \mathbf{I}\right)
$$

Here, $\Gamma$ is the interfacial tension and $\mathbf{q}$ is the interface tensor describing anisotropy of interface. In Eq. (1), $V$ represents the entire volume of the system, $\mathbf{n n}$ is the dyadic of the unit vector n normal to the interface, and I denotes the unit tensor. The integration is made over the entire interface of the system. Once the shape of interface in the deformed state can be obtained, Eq. (1) becomes extremely useful to calculate the interface contribution to the stress. Based on Eq. (1), Okamoto et al. calculated the excess shear stress for typical droplet 
shapes of flat ellipsoid, rod-like shape, dumbbell, and spheroid. ${ }^{11)}$ Recently we evaluated Eq. (1) based on the calculated result of excess shear stress ${ }^{11)}$ and using experimental data of droplet semi-axes for the PIB/PDMS system $^{1,2}$ and a Hydroxypropylcellulose (HPC) solution/ PDMS system, where the HPC solution is in the isotropic state. ${ }^{3)}$ In the HPC/PDMS system the viscosity ratio and the interfacial tension are much larger than those of the PIB/ PDMS system and also the droplet size distribution is much wider. Although the exact distribution of droplet radius should be incorporated in the HPC/PDMS system, Eq. (1) has been found applicable to predict the excess stress of real blend systems. ${ }^{3)}$ In the narrow distribution PIB/PDMS system, only the normalization by the volume-averaged radius was necessary.

The objectives of the present study are: 1) evaluation of the change in the length of semi axes for rod-like and spheroidal droplets based on the Cohen-Carriere theory to obtain the interface tensor component, 2) evaluation of the excess shear stress based on Eq. (1) using the calculated interface tensor component, 3 ) experimental verification of the predictions on the semi axes and the excess shear stress. In Fig. 1, rod-like and spheroidal droplets observed in step strain experiments are schematically shown. The semi-major $(a)$ and semi-minor axes $(b$ and $c$ ) are defined together with the orientation angle $\theta$ to the flow direction $x$. A rod-like droplet is represented by a cylinder with hemispherical caps of radius $r$ at both ends..$^{1,3,5}$

\section{THEORETICAL BACKGROUND}

\subsection{Shape Recovery of Rod-like and Spheroidal Droplets}

For shape recovery of a deformed droplet in a quiescent matrix, Cohen and Carriere ${ }^{5)}$ presented a differential equation

$$
\Gamma \frac{d A}{d L}+6 \pi \chi_{0} \eta_{\mathrm{eff}} r \frac{d L}{d t}=0
$$
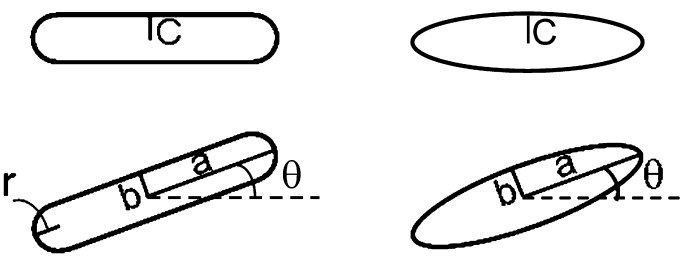

\section{flow}

Fig. 1. Definition of semi-axes $(a, b, c)$, radius $r$ of semi-sphere of endcaps and orientation angle $\theta$ : Top view (upper) and side view (lower). considering a force balance between interfacial tension contribution (first term) and viscous resistance (second term). Here, $A$ is the interfacial area, $L(=2 a)$ the overall length, and $r(=b=c)$ is the radius of rod-like droplet. The time scale of retraction for the stretched droplet is determined by this balance and can be written as

$$
\tau_{\text {ret }}=\frac{\chi_{0} \eta_{\mathrm{eff}} r_{0}}{\Gamma}
$$

where $\chi_{0}$ is a shape factor, $\eta_{\text {eff }}$ is an effective viscosity, and $r_{0}$ is the radius of a sphere having the same volume as the deformed droplet. The factor $\chi_{0}$ was introduced to describe differences in the viscous force arising from differences in droplet shapes. The most plausible form proposed for $\eta_{\mathrm{eff}}$ is

$$
\eta_{\mathrm{eff}}=\eta_{m} \frac{(19 K+16)(2 K+3)}{40(K+1)}
$$

Here, $\eta_{m}$ is the matrix viscosity and $K$ is the viscosity ratio $K=\eta_{d} / \eta_{m}$ with the droplet viscosity $\eta_{d}$. Then $\tau_{\text {ret }}$ becomes $\tau_{\text {ret }}=\chi_{0} \tau_{D}$, where $\tau_{D}$ is the viscoelastic relaxation time of Palierne (emulsion model $)^{12)}$ with a limit of volume fraction $\phi=0$.

$$
\tau_{D}=\frac{\eta_{m} r_{0}}{\Gamma} \frac{(19 K+16)(2 K+3)}{40(K+1)}
$$

In case of a rod-like droplet, Cohen and Carriere ${ }^{5)}$ derived an expression for the normalized radius $R\left(=r / r_{0}\right)$.

$$
\begin{aligned}
& f_{1}(R)-f_{1}\left(R_{R}\right)=\frac{t-t_{R}}{\chi_{0} \tau_{D}} \quad\left(t \geq t_{R}\right) \\
& f_{1}(x)=-x-\frac{8}{x^{2}}+3 \ln \left[\frac{\sqrt{1+x+x^{2}}}{1-x}\right]+3 \sqrt{3} \arctan \left[\frac{\sqrt{3} x}{2+x}\right]
\end{aligned}
$$

Here, $R_{R}$ denotes the value of $R$ at a time $t_{R}$ when the droplet shape becomes rod-like. From the volume conservation requirement, $R$ is obtained by solving the following equation ${ }^{5)}$

$$
R^{3}-3 \lambda R^{2}+2=0
$$

where $\lambda$ is the stretch ratio of semi major axis $a$ (principal stretch), $\lambda=a / r_{0}=(L / 2) / r_{0}$. The analytical solution of Eq. (8) is ${ }^{11)}$

$$
\begin{aligned}
R=\lambda & {\left[1-\cos \left(\frac{1}{3} \arctan \frac{\sqrt{2 \lambda^{3}-1}}{\lambda^{3}-1}\right)\right.} \\
& \left.+\sqrt{3} \sin \left(\frac{1}{3} \arctan \frac{\sqrt{2 \lambda^{3}-1}}{\lambda^{3}-1}\right)\right]
\end{aligned}
$$


The time dependence of $\lambda$ can be obtained by combining Eqs. (6), (7) and (9), when an initial condition $\lambda_{R}=\lambda\left(t_{R}, \gamma\right)$ is given.

Based on the above force balance equation, Okamoto et al. derived the following equations for the shape recovery process from spheroid to sphere under the condition of constant volume $^{4,13)}$,

$$
\begin{aligned}
& f_{2}\left(\frac{1}{\sqrt{\lambda}}\right)-f_{2}\left(\frac{1}{\sqrt{\lambda_{\mathrm{S}}}}\right)=\frac{t-t_{\mathrm{S}}}{24 \chi_{0} \tau_{D}} \quad\left(t \geq t_{\mathrm{S}}\right) \\
& f_{2}(x)=-\frac{1}{2 x^{2}}-\frac{1}{6} \ln \left[\frac{(x-1)^{2}}{x^{2}+x+1}\right]+\frac{1}{\sqrt{3}} \arctan \left[\frac{2 x+1}{\sqrt{3}}\right]
\end{aligned}
$$

where $1 / \sqrt{\lambda}$ is the normalized semi-minor axis, $1 / \sqrt{\lambda}=b / r_{0}$ $=c / r_{0}$, and $\lambda=a / r_{0}$ with $a=L / 2$. In Eq. (10), $t_{S}$ is a time when the droplet shape recovers to spheroid, and $\lambda_{S}$ is the principal stretch at $t=t_{S}$. It was found experimentally that $\lambda_{S}$ is independent of the applied strain $\gamma$ and the average value is $\lambda_{S}=1.49$ for $K=0.067 .^{1,2)}$ A similar, but slightly smaller value of $\lambda_{S}$ is obtained for $K=0.54 .^{3,14)}$ On the other hand, the time $t_{S}$ increases with $\gamma$ due to the delayed shape recovery at large $\gamma$. As shown in Eqs. (10) and (11), the retraction time $\chi_{0} \tau_{D}$ also governs the shape recovery from spheroid to sphere.

In Eqs. (6) and (10), $\chi_{0}$ is a hydrodynamic factor originally introduced by Cohen and Carriere. ${ }^{5)}$ An anonymous reviewer kindly pointed out that in principle, $\chi_{0}$ is determined by flow mode (distribution of shear and elongation fields) in the droplet retraction process and that $\chi_{0}$ is not an adjustable parameter. We thank the reviewer for the pertinent comment on $\chi_{0}$. Cohen and Carriere introduced $\chi_{0}$ based on the hydrodynamic calculation by Lamb ${ }^{15)}$ that the resistance experienced by a circular disk with radius $c$ moving in a liquid of viscosity $\eta$ becomes $6 \pi \eta r u$. Here, $u$ is the velocity and $r$ is the effective radius expressed as $r=0.850 c$ for broadside-on motion and $r=0.566 c$ for edgewise motion. Since $r$ does not alter its geometrical meaning for the significantly different motions, the viscous resistance to the fiber retraction was assumed as $6 \pi \chi_{0} \eta_{\text {eff }} r u$ in the Cohen-Carrier theory. In a quiescent matrix, $u$ is replaced by $u=d L / d t$. For deformable viscous droplets, a few forms of $\eta_{\text {eff }}$ were presented, all of which are proportional to $\eta_{m}$ and similar in value for $\mathrm{K}<10^{5,17-19)} \mathrm{In}$ their treatment, the factor $\chi_{0}$ is always determined by comparison between the prediction (Eqs. (6) and (7)) and experimental data. Values obtained from this comparison for rod-like droplets by Cohen and Carriere are $\chi_{0}=3.5 / 6 \pi=0.186^{16)}$ and $1 /(2 \times 2.7)=0.185^{18)}$, where a factor 2 in the latter expression is due to the difference in definition of $f_{1}(x)$.
On the other hand, Okamoto et al. ${ }^{4)}$ proposed $\chi_{0}=1 / 8=0.125$ for spheroidal droplets from the following argument. In the final process of droplet retraction, $1 / \sqrt{\lambda}$ approaches 1 , and $f_{2}(x)$ can be approximated to be

$$
f_{2}(x) \cong-\frac{1}{3} \ln (1-x)-\frac{1}{2}+\frac{1}{6} \ln 3+\frac{\pi}{3 \sqrt{3}}
$$

From Eqs. (10) and (12), we obtain

$$
1-\frac{1}{\sqrt{\lambda}} \cong C \exp \left(-\frac{t}{8 \chi_{0} \tau_{D}}\right)
$$

with a constant $\mathrm{C}$

$$
C=\left(1-\frac{1}{\sqrt{\lambda_{S}{ }^{\prime}}}\right) \exp \left(\frac{t_{S}^{\prime}}{8 \chi_{0} \tau_{D}}\right)
$$

where $t_{S}^{\prime}\left(>t_{S}\right)$ and $\lambda_{S}{ }^{\prime}\left(=\lambda\left(t_{S}{ }^{\prime}\right)<\lambda_{S}\right)$ are taken in the range where Eq. (12) can be applied. From Eq. (13), the Hencky strain $\ln \lambda$ for spheroidal droplet in the final process is approximated to be

$$
\ln \lambda \approx 2 C \exp \left(-\frac{t}{8 \chi_{0} \tau_{D}}\right)
$$

The experimental data of $\ln \lambda$ in the final process ${ }^{1,2}$ approximately have this form with a time constant equal to $\tau_{D}$ Thus $8 \chi_{0}$ becomes 1 , giving $\chi_{0}=1 / 8$. Here, for simplicity let us consider small deformation limit. There is only one time constant $8 \chi_{0} \tau_{D}$ for shape recovery from slightly oblate spheroid to sphere due to the interfacial tension. The viscoelastic relaxation time $\tau_{D}$ associated with this process (interfacial-tension origin) should be equal to (or at least very close to) $8 \chi_{0} \tau_{D}$. Thus $\chi_{0}$ should be $1 / 8$ or around $1 / 8$. A simple exponential form, Eq. (15), is only approximate and may be valid in the small deformation limit.

It should be noted here that the total recovery time after application of a large step strain becomes longer with increasing $\gamma$, because both times $t_{R}$ and $t_{S}$ increase with $\gamma$. However, Eq. (10) with constant $\lambda_{S}$ suggests that the shape recovery after $t_{S}$ from spheroid to sphere occurs at the same rate ( $\gamma$-independent rate) and that $\lambda$ is the same for the same interval $\left(t-t_{\mathrm{S}}\right) / \tau_{D}$, irrespective of $\gamma$ applied.

\subsection{Excess Shear Stress for Rod-like and Spheroidal Droplets}

We define the volume fraction $\phi$ for a single droplet with the radius $r_{0}$ by $\phi=\left(4 \pi r_{0}^{3} / 3\right)(1 / V)$. From Eq. (1), the excess shear stress $\Delta \sigma_{x y}$ can be written as 


$$
\Delta \sigma_{x y}=-\frac{3 \Gamma \phi}{4 \pi r_{0}^{3}} \int d S n_{x} n_{y}
$$

For a rod-like droplet shown in Fig. 1 (or for monodisperse rod-like droplets), $\Delta \sigma_{x y}$ becomes

$$
\Delta \sigma_{x y}=\left(\frac{3 \Gamma \phi}{8 r_{0}}\right) \sin 2 \theta[2(\lambda-R) R]
$$

with the principal stretch $\lambda\left(=a / r_{0}\right)$ and $R=b / r_{0}=c / r_{0}=r / r_{0}$. In a step strain experiment, a deformed droplet becomes spheroid just before returning to a spherical shape, irrespective of the magnitude of strain applied. For monodisperse spheroidal droplets having a common orientation angle $\theta$, the analytical solution of the excess shear stress $\Delta \sigma_{x y}$ becomes ${ }^{2,11}$

$$
\begin{aligned}
\Delta \sigma_{x y}= & \left(\frac{3 \Gamma \phi}{8 r_{0}}\right) \sin 2 \theta \\
& {\left[\frac{\lambda^{3}+2}{\lambda\left(\lambda^{3}-1\right)}+\frac{\sqrt{\lambda}\left(\lambda^{3}-4\right)}{\left(\lambda^{3}-1\right)} \frac{\arcsin \sqrt{1-\lambda^{-3}}}{\sqrt{1-\lambda^{-3}}}\right] }
\end{aligned}
$$

with $\lambda=a / r_{0}$ and $b / r_{0}=c / r_{0}=\lambda^{-1 / 2}$.

When the orientation angle $\theta$ is equal to an angle given by affine deformation assumption and does not change with time, $\theta$ satisfies the following equation

$$
\cot 2 \theta=\gamma / 2
$$

It has been found in stress relaxation experiments for step strain that Eq. (19) holds for droplets with the viscosity ratio $K=0.067^{1,2)}$ and $K=0.0476 .{ }^{13)}$ Very recently, we found that Eq. (19) also holds for droplets with $K=0.54 .^{3,14)}$

\section{MATERIALS AND METHOD}

Observation of deformation and shape recovery of PIB droplets in a PDMS matrix under large step shear strains is already reported in the previous papers. ${ }^{1,2)}$ The weight-average molecular weight of PIB is 1350, and the zero shear viscosities of PIB $\left(\eta_{d}\right)$ and PDMS $\left(\eta_{m}\right)$ at $23{ }^{\circ} \mathrm{C}$ are 60 and 900 Pas, respectively. The viscosity ratio $K$ is 0.067 . The interfacial tension obtained by the pendant drop method for this system is $\Gamma=3.1 \times 10^{-3} \mathrm{~N} / \mathrm{m}^{2,6)}$ The semi-axes $a$ and $b$ were observed from the side view and $c$ was obtained from the top view. New reliable data for a droplet with $r_{0}=140 \mu \mathrm{m}$ are added in the present study. The relaxation time $\tau_{D}$ calculated by Eq. (5) is $\tau_{D}=51.5$ and $84.5 \mathrm{~s}$ for droplets with $r_{0}=140$ and $230 \mu \mathrm{m}$, respectively.
For a 20/80 (wt/wt) blend of PIB/PDMS, experimental data of stress relaxation under large step shear strains at $23{ }^{\circ} \mathrm{C}$ are already reported. ${ }^{11)}$ Decomposition of the blend modulus into component and interface contributions has been made and is published elswhere. ${ }^{3)}$ Dynamic viscoelastic data of the blend and components were obtained, and decomposition into component and interface contributions has been done and is reported. ${ }^{3)}$ To obtain reproducible data with the same distribution of droplet radius, pre-shear at the strain rate of $0.25 \mathrm{~s}^{-1}$ for $1240 \mathrm{~s}$ has been given to the blend samples before dynamic measurements, ${ }^{3)}$ as already done in stress relaxation experiments. ${ }^{11)}$ After the pre-shear, the volume-averaged radius $r_{V}$ was found to be $r_{V}=9.0 \mu \mathrm{m}$, and the distribution of droplet size was rather narrow, $r_{V} / r_{N}<1.2$, where $r_{N}$ is the number-averaged radius., ${ }^{3,11)}$ The relaxation time $\tau_{D}$ of the blend with $\phi=0.214$ becomes $4.1 \mathrm{~s}$, which is evaluated by

$$
\tau_{D}=\frac{\eta_{m} r_{V}}{4 \Gamma} \frac{(19 K+16)[2 K+3-2 \phi(K-1)]}{10(K+1)-2 \phi(5 K+2)}
$$

\section{RESULTS AND DISCUSSION}

\subsection{Retraction of Semi Major Axis for Rod-like and Spheroidal Droplets}

In Fig. 2, the prediction of time dependence of the principal stretch, $a / r_{0}$, for a rod-like droplet is compared with experimental data at $\gamma=3-5$ for droplets with $r_{0}=230$ and $140 \mu \mathrm{m}$. A comparison at $\gamma=2$ is omitted, because the rod-like shape appears in very limited time scale at that strain, ${ }^{1)}$ and exact comparison of the time dependence is impossible. For $\chi_{0}=0.175$, the theoretical prediction by Cohen-Carriere agrees

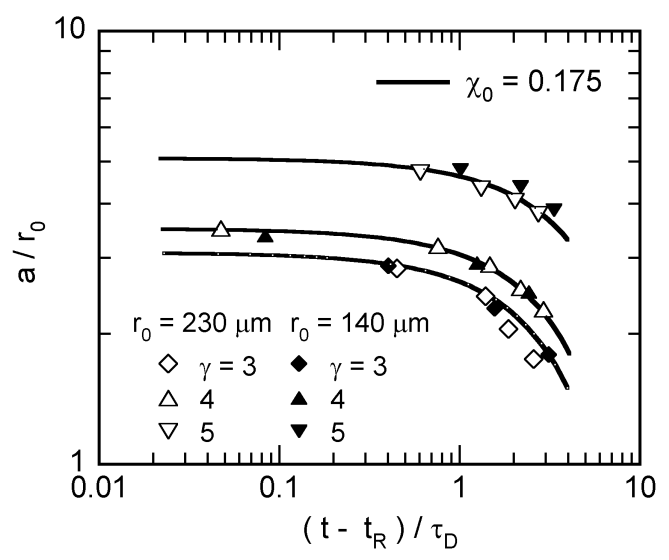

Fig. 2. The stretch ratio of semi-major axis as a function of normalized time in the intermediate stage of relaxation with rod-like shape. $\chi_{0}$ is a hydrodynamic factor originally introduced by Cohen and Carriere. 
fairly well with experimental data. The value of $\chi_{0}=0.175$ obtained in the present study is essentially the same as that obtained by Cohen-Carrier, $\chi_{0}=0.185-0.186$. Considering the experimental error of about $12 \%$ in the interfacial tension $\Gamma^{6)}$ and somewhat greater experimental error in $\tau_{D}$ due to accumulated errors in $\Gamma, \eta_{m}$ and $r_{0}, 6 \%$ difference in $\chi_{0}$ is minor. The initial stretch $\lambda_{R}$ and the time $t_{R} / \tau_{D}$ determine the subsequent shape recovery in this process. In Fig. 3 the strain dependencies of $\lambda_{R}$ and $t_{R} / \tau_{D}$ are shown, which are obtained experimentally at $\gamma=2-5$. The strain dependence of $t_{R}$ is weak, say $t_{R} \sim \gamma^{0.54}$. It seems that $\lambda_{R}$ and $t_{R} / \tau_{D}$ at each strain are independent of the initial droplet size $r_{0}$ as shown in Fig. 3.

In Fig. 4, the prediction of time dependence of the principal stretch, $a / r_{0}$, for spheroid is compared with experimental data at $\gamma=1-5$ for droplets with $r_{0}=230$ and $140 \mu \mathrm{m}$. For $\chi_{0}=0.110$, very good agreement with the experimental data is obtained for all the strains measured. Note that the ordinate is enlarged to emphasize the agreement. The value of $\chi_{0}=0.110$ is very close to the value $\chi_{0}=0.125$ predicted by Okamoto $e t$ $a l .{ }^{4)}$ (13.6\% difference), considering the experimental error of about $12 \%$ in $\Gamma$ and accumulated experimental error in $\tau_{D}$. It becomes clear that the shape recovery in the final stage can be described by specifying the initial values $\lambda_{S}(=1.49)$ and $t_{S} / \tau_{D}$ of this stage. The effect of previous shape (memory) does not appear in the time dependence at $t>t_{S}$. The strain dependence of $t_{S} / \tau_{D}$ obtained experimentally for these droplets is shown in Fig. 3. The strain dependence of $t_{S}$ is rather strong, say $t_{S} \sim \gamma^{1.8}$. This strain dependence is attributed to the delayed shape recovery in the early and intermediate stages of relaxation. It seems that $t_{S} / \tau_{D}$ at each strain is also independent of $r_{0}$ as shown in Fig. 3.

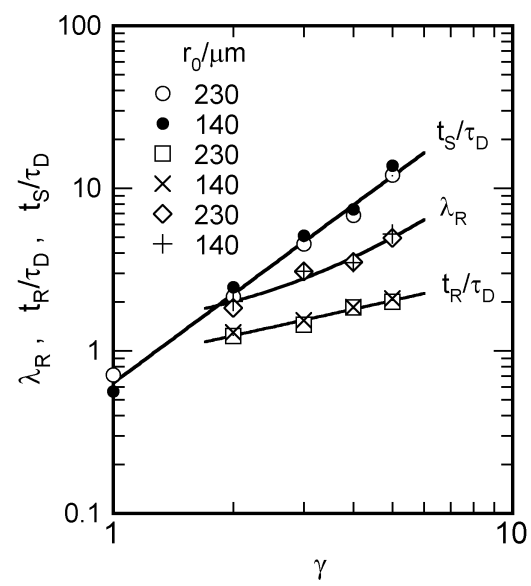

Fig. 3. Strain dependencies of the characteristic times $t_{R}$ and $t_{S}$ and of the principal stretch $\lambda_{R}$ at $t_{R}$, where the subscripts $\mathrm{R}$ and $\mathrm{S}$ denote rodlike shape and spheroid.

\subsection{Excess Shear Stress for Rod-like and Spheroidal Droplets}

Time dependences of normalized $\Delta \sigma_{x y}$ in the recovery process with the rod-like shape are summarized in Fig. 5. The excess shear stress obtained for the $20 / 80$ blend is normalized by replacing $r_{0} / \Gamma$ with $r_{V} / \Gamma$. When the same value of $\chi_{0}=0.175$ as in Fig. 2 is used, the agreement between the theoretical prediction and the experimental data is fair at short and intermediate time scale of $\left(t-t_{R}\right) / \tau_{D}$. However, at longer time scale of $\left(t-t_{R}\right) / \tau_{D}$, the excess shear stress of the blend relaxes faster than the prediction. Three possibilities for the discrepancy may be considered. The first is uncertainty in $t_{R} / \tau_{D}$ for the polydisperse droplet system. The second is attractive interaction between adjacent droplets which may result in faster recovery in the blend than the single-droplet recovery (see next paragraph). The third is reduction of hydrodynamic interaction in concentrated blend, because a smaller value of $\chi_{0}$ such as 0.110 seems to describe better the experimental data as shown by dotted lines in Fig. 5. However, the third possibility is not decisive, considering somewhat large experimental error in $\Gamma$ and $\tau_{D}$.

Okamoto et al. observed collision and coalescence of two droplets on the same plane (parallel to the shearing plane) in an immiscible matrix after application of large step strains. ${ }^{20,21)}$ In case of shape recovery without coalescence, the droplets come closer with each other after recovery due to an attractive interaction during retraction process. This attractive interaction is considered to be hydrodynamic origin (hydrodynamic tensor form). The coalescence depends on the applied strain and the initial distance $d_{0}$ between the centers of two droplets, or more precisely on $d_{0} / r_{0}{ }^{20)}$ In fact, two droplets coalesce into

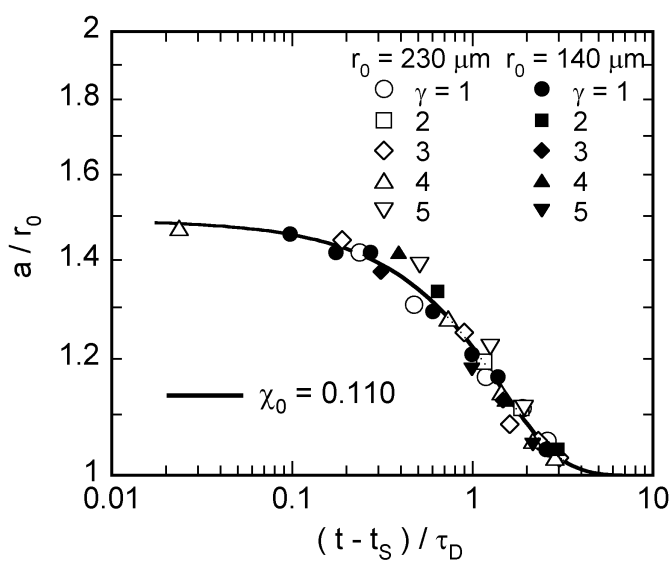

Fig. 4. The stretch ratio of semi-major axis as a function of the normalized time in the final stage of relaxation. $\chi_{0}$ is a hydrodynamic factor originally introduced by Cohen and Carriere. 
one droplet after repetition of large step strains in the opposite directions or accumulated strains. ${ }^{21)}$ It is probable that the attractive interaction also occurs in the blend, because average value of $d_{0} / r_{0}$ is rather small $\left(d_{0} / r_{0}=2.69\right)$ in the 20/80 blend with $\phi=0.214$ assuming the simple cubic lattice for the droplet distribution. For PIB droplets in a PDMS matrix with very similar viscosities with those of the present study, Okamoto et $a l$. found in a single step test that at $\gamma=4.58$ two droplets recover to the spherical shape without coalescence for $d_{0} / r_{0}=2.68$, but coalescence occurs for $d_{0} / r_{0}=2.29 .{ }^{20)}$ Overlap of two droplets is observed in both cases with and without coalescence, and in the overlapped part the hydrodynamic attractive force may work between the droplets (or between the interface). The hydrodynamic force is considered to arise when the local matrix flow occurs due to the change in the droplet shape. ${ }^{20)}$ On the other hand, in the un-overlapped part, the interfacial tension force acts to recover to the original shape. Normal vectors to the interface become isotropic due to the interfacial tension. ${ }^{20)}$

The excess shear stress normalized as $\gamma$-independent form in the final stage of relaxation with the spheroidal shape is shown in Fig. 6. For the stress prediction in this process, the same value of $\chi_{0}=0.110$ is used as in Fig. 4 . We can see from Fig. 6 that experimental data of normalized $\Delta \sigma_{x y}$ are almost independent of $\gamma$ as expected from the theoretical prediction. However, closer look at the data reveals that the time dependence of normalized stress becomes somewhat weaker with increasing the strain. This apparent difference in the time dependence is attributed to the effect of polydispersity in droplet size. A variety of deformed shapes and difference in relaxation stage are enhanced with the applied strain.

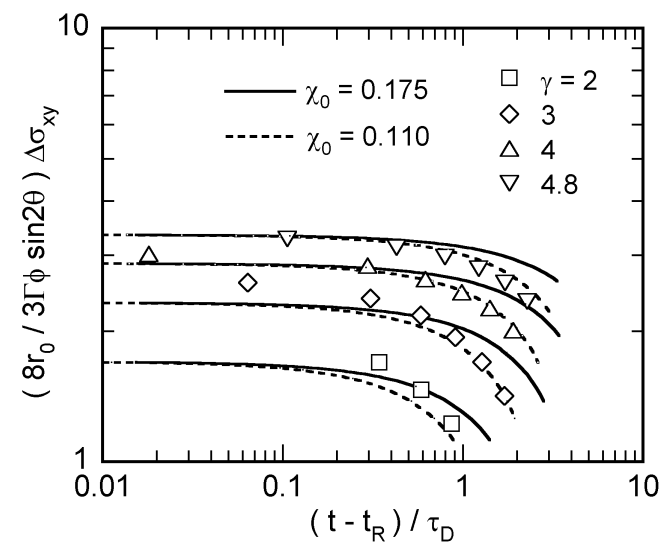

Fig. 5. Predicted time dependence of the normalized excess shear stress in the intermediate stage of relaxation with rod-like shape is compared with experimental data for a 20/80 blend of PIB/ PDMS. The data are normalized per one droplet with the volumeaveraged radius.
Scattering of the data at shorter time scale of $\left(t-t_{S}\right) / \tau_{D}$ is also attributed to the uncertainty of $t_{S} / \tau_{D}$ in polydisperse droplet system. In this final stage of shape recovery, interaction between adjacent droplets is reduced, because small droplets have already recovered to the spherical shape. The effect of droplet size distribution is considered to be more important than the interaction with adjacent droplets or change in hydrodynamic interaction in the final stage.

\section{CONCLUSIONS}

The Cohen-Carriere prediction on retraction of semi axes for a rod-like droplet in an immiscible matrix is compared with experimental data on a poly(isobutylene) (PIB) droplet in a poly(dimethyl siloxane) (PDMS) matrix. Satisfactory agreement between the prediction and the experimental data is obtained when a shape factor (hydrodynamic factor) $\chi_{0}$ is 0.175 . The prediction presented by Okamoto et al. applying the Cohen-Carrier theory to spheroidal droplet is compared with experimental data of the same system. Very good agreement between the prediction and the data is obtained when $\chi_{0}$ is 0.110 . These values of $\chi_{0}$ are essentially the same as (or very close to) those suggested by Cohen-Carrier $\chi_{0}=0.185-0.186$ for a rod-like droplet and by Okamoto et al. $\chi_{0}=0.125$ for a spheroidal droplet.

The excess shear stress or interfacial contribution to the shear stress is evaluated based on the Doi-Ohta theory using the calculated droplet dimensions for rod-like and spheroidal

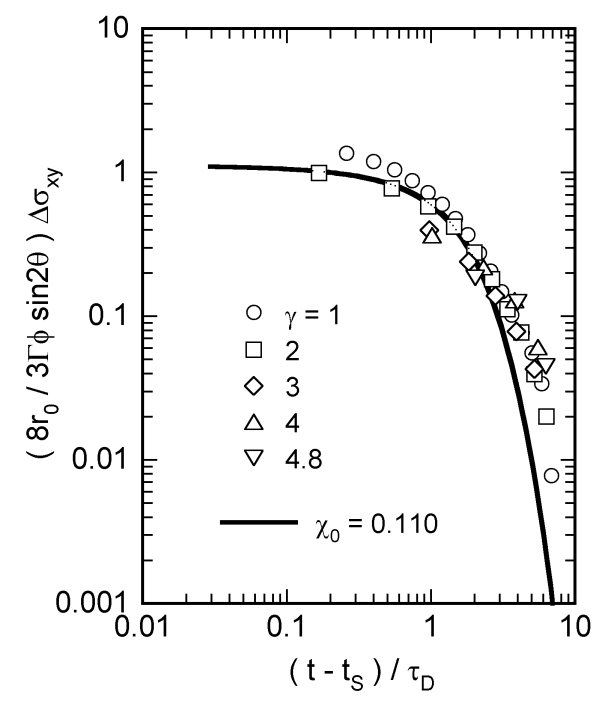

Fig. 6. Predicted time dependence of the normalized excess shear stress in the final stage of relaxation is compared with experimental data obtained for a 20/80 blend of PIB/PDMS. The data are normalized per one droplet with the volume-averaged radius. 
droplets. The predicted excess shear stress is compared with experimental data for a 20/80 blend of PIB/PDMS after normalization per single droplet with the volume-averaged radius. In the rod-like stage, the predicted stress agrees fairly well with experimental data at short and intermediate time scales but fails at longer times. Three possibilities (polydisperse effect, attractive interaction between adjacent droplets and reduction in $\chi_{0}$ ) are considered for the discrepancy at longer time scale. The attractive interaction is shown to be most probable. In the final stage with spheroidal shape, the predicted stress is close to the experimental data. However, the time dependence of the experimental stress data becomes weaker with increasing the step strain. It is suggested that the effect of droplet size distribution is more important than the dropletdroplet interaction or change in $\chi_{0}$ in the final stage.

\section{Acknowledgments}

This work was partially supported by Grant-in-Aid for Scientific Research (B) No. 16350127 and 18350119 from the Japan Society for the Promotion of Science.

\section{REFERENCES}

1) Yamane H, Takahashi M, Hayashi R, Okamoto K, Kashihara H, Masuda T, J Rheol, 42, 567 (1998).

2) Hayashi R, Takahashi M, Yamane H, Jinnai $H$, Watanabe $H$, Polymer, 42, 757 (2001).
3) Takahashi M, Macaúbas PHP, Okamoto K, Jinnai H, Nishikawa Y, Polymer, 48, 2371 (2007).

4) Okamoto K, Takahashi M, Yamane H, Watashiba H, Tsukahara Y, Masuda T, Nihon Reoroji Gakkaishi (J Soc Rheol Japan), 27, 109 (1999).

5) Cohen A, Carriere CJ, Rheol Acta, 28, 223 (1989)

6) Hayashi R, Takahashi M, Yamane H, Nihon Reoroji Gakkaishi (J Soc Rheol Japan), 28, 137 (2000).

7) Batchelor GK, J Fluid Mech, 41, 545 (1970).

8) Onuki A, Phys Rev A, 35, 5149 (1987).

9) Onuki A, Europhysics Lett, 28, 175 (1994).

10) Doi M, Ohta T, JChem Phys, 95, 1242 (1991).

11) Okamoto $K$, Takahashi $M$, Watanabe $H$, Koyama $K$, Masuda $T$, Proceedings of the International Conference on Advanced Polymers and Processing (Yamagata), 195 (2001).

12) Palierne JF, Rheol Acta, 29, 204 (1990).

13) Okamoto K, Takahashi M, Yamane H, Kashihara H, Watanabe H, Masuda T, J Rheol, 43, 951 (1999).

14) Macaúbas PHP, Kawamoto H, Takahashi M, Okamoto K, Takigawa T, Rheol Acta, in press.

15) Lamb H, "Hydrodynamics”, (1945), Dover, New York.

16) Cohen A, Carriere CJ, Rheol Acta (Erratum), 28, 435 (1989).

17) Carriere CJ, Cohen A, Arends CB, J Rheol, 33, 681 (1989).

18) Carriere CJ, Cohen A, J Rheol, 35, 205 (1991).

19) Rundqvist T, Cohen A, Klason C, Rheol Acta, 35, 458 (1996).

20) Okamoto K, Tamura R, Ishikawa M, Nihon Reoroji Gakkaishi (J Soc Rheol Japan), 30, 45 (2002).

21) Okamoto $\mathrm{K}$, Iwatsuki $\mathrm{S}$, Osaki $\mathrm{K}$, Ishikawa $\mathrm{M}$, Proc XIVth Int Congr Rheology (Seoul), PM57-1 (2004). 